\title{
Nous veillerons ensemble sur le sommeil des hommes de Françoise Lalande : poétique et poétisation du corps
}

\author{
Mariem Lahmar \\ Faculté des Lettres, des Arts et des Humanités de la Manouba
}

\section{Introduction}

«Je sais pour qui j'écris. D'abord pour moi, pour ramener de mes abîmes intimes ce qui me tourmente, m'intrigue, me fait parfois souffrir, me met dans des états terribles ", révèle Françoise Lalande dans une interview accordée à Jeannine Paque dans la revue Le Carnet et les Instants en mars 2012.

Née en Ardennes, le 2 octobre 1941, donc en pleine Seconde Guerre mondiale, Lalande s'imprègne de cette situation tragique et s'en inspire afin d'écrire ses œuvres. Cette écrivaine fait en effet de l'enfance avortée et tronquée un thème prédominant dans ses écrits. La douleur, la séparation et l'horreur de la guerre sous-tendent nombre de ses récits qui illustrent la folie assassine de l'homme, dans un monde en crise.

En écrivant, l'auteure affirme vouloir se libérer d'elle-même et de ce passé vécu dans la peur et dans la fuite. Gardant en mémoire l'histoire qui a douloureusement bercé et bouleversé son enfance, Lalande se porte comme témoin de tout ce qui tourmente, fait souffrir et horrifie. Thème sous-jacent, l'histoire irrépressible est ce qui jalonne la ligne directrice de tous les écrits de cette écrivaine. Ses personnages sont les figures d'une force humaine qui surmonte la douleur d'une manière ou d'une autre. Lalande adopte dans ses écrits un style d'écriture bien particulier et fait du silence une occasion pour donner libre cours au corps afin qu'il s'y exprime, apte à remplacer la parole. Une écriture qui se veut libératrice et annonciatrice à la fois, qui libère de tout ce qui est reçu comme certitude et appelle au doute, qui convie à repenser et à revisiter ce qui se prend pour intouchable.

Dans cette conception de l'écriture, le corps, chez Lalande, occupe donc un rang important et joue un rôle central. Sa manifestation, qui ne peut qu'interpeller directement le lecteur, nous renvoie surtout à sa représentation dans son chef-d'œuvre intitulé Nous veillerons ensemble sur le sommeil des hommes (2012). Le lecteur y assiste à une mise en scène fort originale du corps et où celui-ci est complètement mis à nu, peint tel qu'il est, dans tous ses états et toute sa beauté. Rattachée au thème de la souffrance, cette nouvelle image corporelle se déploie en correspondance avec des images mythiques riches en significations. 
Le roman comprend trois histoires séparées, celles de Léa, de Lila et de Julius Keil, lesquels se rencontrent à la fin de l'histoire lors d'une réunion juive rassemblant en Israël toutes les personnes portant leur nom et venues de tous les coins du monde. Chaque histoire débute avec l'enfance du héros vécue, pour les deux femmes, pendant la Seconde Guerre, à l'époque de l'oppression exercée par les Allemands antisémites. Léa passe la première partie de son enfance avec sa mère Myriam-la-jeune à Berlin. Après le décès de celle-ci, elle est placée dans un orphelinat. C'est à partir de là qu'elle devient une jeune musicienne douée. Lila, elle, passe son enfance chez son grand-père en Belgique avec sa mère et son beau-père. Adolescente, elle quitte sa famille et intègre l'université avant de devenir écrivaine et lutteuse professionnelle. Quant à Julius, orphelin de père depuis son jeune âge, il grandit à Seattle avec une mère possessive et sévère. Il hérite la passion de son père et choisit d'être marin. Dès le début de chacune de ces destinées, nous assistons à la mise en place de toutes sortes de cicatrices ayant douloureusement marqué l'enfance de chaque protagoniste. Une biographie grâce à laquelle, au fur et à mesure que se tisse la trame narrative, le lecteur peut suivre les états psychologiques des héros et l'évolution de leur personnalité. C'est dans ce contexte que l'auteure joint corps et souffrance pour crier haut et fort son «malaise et [sa] nausée face à l'espèce humaine capable d'accomplir ça » (Paque 2012).

\section{Un corps poétisé}

Écrire le corps, en exprimer les manifestations, dépend de la manière dont on le conçoit. En effet, le travail de l'écriture réfléchit la pensée de celui qui écrit sous forme d'images que le lecteur peut se représenter à partir du tissage des mots. L'image du corps qui est représentée dans ce roman est avant tout celle d'un corps épanoui et qui se libère. Au milieu de l'atmosphère de désespoir et d'accablement que les personnages subissent à cause de l'histoire, des cicatrices de la guerre et des séquelles ancestrales, se trouve en effet un corps qui combat, se surpasse et tente de surgir d'entre les décombres, tout comme Vénus surgit du milieu de l'Océan.

Grâce à l'échange érotique, les protagonistes retrouvent goût à la vie. Le corps leur permet de renouer avec elle lorsqu'ils sont sur le point de succomber au désespoir. L'activité sexuelle permet non seulement de renouer avec les plaisirs quotidiens, mais elle éveille aussi les sens et ouvre une nouvelle brèche afin de pouvoir dépasser la douleur. Myriam-la-jeune, la mère de Léa Keil, en est un exemple éloquent. Avant la rencontre avec le jeune homme inconnu, n'avait-elle pas oublié sa féminité? Dans cette guerre où les Allemands persécutent les juifs, Myriam mène en effet une vie 
misérable avec sa petite fille chez de vieux Allemands qui leur ont donné refuge ${ }^{1}$. Leur vie semble n'avoir qu'un seul but : survivre.

Le soir venu, Myriam-la-jeune contemplait de sa fenêtre le paysage dévasté [...], la ville n'était plus qu'un souffle, qui se faufilait d'une fenêtre à l'autre, glissant d'un immeuble à l'autre, n'épargnant rien ni personne, agressant les cerveaux, remodelant les corps, le grand vent nettoyeur, rapide comme une vipère, qui charriait les pestilences du passé pour les emporter loin de la ville (15-16).

La ville allemande décrite au début du roman, Berlin, est une ville fantôme qui donne l'impression d'étouffer. Le regard du personnage qui constate tristement les multiples dégâts reflète ce sentiment d'asphyxie. Ce «souffle» ravageur résulte de l'observation de cette ville ravagée, que Myriam scrute sans pouvoir s'empêcher de penser à sa propre condition tragique. La protagoniste s'identifie à cette ville anéantie, sans âme ni corps. «Mon squelette ruiné est à l'image de cette ville » (30). Femme forte malgré tout, elle devient «remblayeuse de décombres» (19) et se donne corps et âme à la reconstruction de la ville en compagnie d'autres femmes berlinoises. L'oubli de sa féminité la pousse à se couper les cheveux, un geste conçu par elle-même comme un acte de coquetterie alors que traditionnellement, la beauté d'une femme est liée à sa chevelure. Toutefois, ce désir est vite interrompu par la peur qu'elle éprouve de rendre son humeur encore plus «désastreuse ». Myriam renvoie alors cette tentation sur sa fille. Ainsi, la féminité est-elle non seulement absente, mais également accentuée par une sorte de castration que cette femme effectue sur son propre corps et sur celui de sa fille, de manière inconsciente : «Myriam-la-jeune, plongée jusqu'au cou dans la boue, jugea indécent de céder à la coquetterie si bien qu'elle renonça à se couper les cheveux ce jour-là, mais elle reporta, ainsi que le font souvent les mères, son désir de changement sur sa fille, et, sans lui demander son avis, elle coupa les cheveux de Léa » (20).

Le renoncement à la féminité, qui passe automatiquement par une mise à mal du corps, entraîne la protagoniste dans une série de crises existentielles : réfléchir sur le monde, sur la situation de l'être humain face aux atrocités qu'il a commises, sur les juifs et leur douleur ainsi que sur les Allemands qui n'ont eu de cesse de les massacrer. Myriam, oubliant qu'elle est une femme, oublie en même temps de prendre soin de son corps et s'adonne au travail comme si elle était un homme : "elle enfilait un pantalon de toile, trop large, qu'elle retenait à la taille à l'aide d'une corde, elle chaussait des bottines d'hommes, et elle descendait dans la rue, rejoindre d'autres femmes attifées comme elle, Salut les épouvantails à moineaux!» (14). Son corps ne respire que lorsque «quelque chose de différent arriva, une nouveauté dans l'air, à moins que ce ne fût dans le corps » (21). La jeune femme a si

\footnotetext{
${ }^{1}$ L'histoire se passe à Berlin en mai 1945. Léa et sa mère Myriam, après la guerre, sont cachées par un vieux couple allemand dans une cave. Touché par leur détresse, il prend le risque de les abriter et partage avec elles tous les détails de son quotidien.
} 
bien hiberné son corps que la rencontre de l'adolescent ${ }^{2}$ éveille ses sens de manière violente et intensive : "elle s'enflamma à son tour, découvrant en un éclair à quel point elle brûlait de faire l'amour, à quel point son corps n'en pouvait plus d'attendre»(22). Dès cette révélation, Myriam prend enfin conscience que son corps a besoin d'être comblé non pas par ses mains de femme, mais par une présence masculine qui accomplirait jusqu'à son terme la forme de plaisir recherchée. Myriam, qui «éprouvait la désagréable sensation d'être une femme transparente, eh bien! Aujourd'hui, elle n'avait pas été transparente puisqu'un homme avait remarqué son corps, désirait le dévorer » (23), comprend combien sa féminité passe par son corps à elle et exige la présence d'un corps accepté et reconnu. Cette rencontre lui permet de « renouer avec le plaisir d'être une femme » (23) et de voir son corps dans toute sa splendeur. En effet, la jeune femme procède par la suite à tout un rituel féminin mettant en exergue un corps pleinement assumé : elle prend soin de sa toilette et prête attention aux plus petits détails avant d'aller à son rendez-vous; elle se fait belle en enfilant des vêtements qui soulignent la beauté d'un corps jusqu'alors enfoui. Une transformation qui ne laisse pas indifférent son compagnon et qui lui permet à elle de vivre sa nouvelle féminité à pleines dents: «il remarqua qu'elle portait des chaussures à semelles compensées et comprit que c'était pour lui plaire que cette inconnue s'était transformée, qu'elle s'était crue chenille et se voulait papillon » (25). La métaphore employée par le narrateur révèle l'ampleur de la métamorphose. Bien que le jeune homme l'ait remarquée dans son naturel, c'est-à-dire dans un état de femme à l'apparence délaissée, il « fut ému par la fragilité de Myriam-la-jeune » (25).

Dans la première scène sexuelle décrite entre Myriam et l'adolescent, les verbes de perception et de sensation abondent et se répètent dans un paragraphe d'une trentaine de lignes : sentir, apercevoir, regarder, voir, observer, caresser, effleurer... La première à remarquer le changement de Myriam est cependant sa fille Léa, car ce premier contact physique avec l'homme inconnu redonne à la jeune femme l'appétit de vivre. Ces verbes de perception montrent à quel point l'épanouissement du corps peut être un moyen de libération, une sorte de charge positive qui donne aux personnages un nouvel élan vers la recherche de soi. Myriam-la-jeune était perdue, elle ne se retrouvait pas dans son corps de femme, qui plus est, elle l'avait oublié et l'enfouissait dans des vêtements amples et nonchalants. Sa condition de femme vivant mal sa féminité l'avait poussée à se détourner d'elle-même, à se tourner uniquement vers les autres (le couple de vieux Allemands et sa fille) et ce qui l'entourait (la guerre et ses atrocités). Mais la découverte de son corps, «un corps en chaleur» qui a besoin d'être caressé et mené jusqu'au comble du plaisir, lui insuffle désormais l'envie d'être une femme à part entière.

${ }^{2}$ Un jeune homme qu'elle a rencontré aux décombres et qu'elle ne connaît pas. 
«Écouter du piano, assister à un concert, rester immobile, perdue au monde, mais entrée dans la musique, cela c'est un luxe que Myriam-la-jeune aurait aimé s'offrir » (32). Grâce à la découverte de son corps, la jeune femme devient en effet plus sensible. Elle qui a toujours été ferme dans sa décision de ne jamais tomber amoureuse, et qui était souvent de mauvaise humeur, prête désormais attention aux détails quotidiens qui la rattachent à la vie. Ainsi la résurrection de Myriam se fait-elle par un corps qui se libère et qui se déploie dans l'activité sexuelle partagée. Elle est telle une Vénus qui émerge des décombres de sa ville en ruine et qui renait entre les pierres et les gravats de la guerre.

Pour sa fille Léa aussi, le corps constituera un lieu de libération lui offrant un nouveau souffle capable de renouveler son énergie. Elle transmettra à son public le même sentiment de plaisir corporel qu'elle partage avec David, son frère de combat, et avec ses amants. L'échange sexuel chez Léa est comme une sorte de rituel indispensable à la préservation de sa passion. Personnage assez particulier dans le roman, elle est présentée comme une fille d'exception, une artiste de génie qui vit de et pour la musique qu'elle compose et qui cherche à communiquer à ses spectateurs «l'amour du monde ». Pour ce faire, il lui faut expérimenter cet amour afin de mieux le comprendre. Sa musique est une musique érotique qui traduit l'épanouissement d'un corps ouvert, prêt à jouir et chantant le plaisir: "Recevoir les caresses puissantes de la musique, comme une jouissance inavouée, le bonheur terrible du désir» (40). Si Léa se fait caresser par ses amants, elle caresse elle aussi son violoncelle pour que son public se sente à son tour caressé par la musique qu'elle produit. Dans ce sens, c'est le corps de Léa qui confère à sa musique toute sa dimension sublime: "Elle parle d'amour par la musique, de la délivrance des cœurs par la voix humaine du violoncelle, de l'émotion érotique des corps que celui-là provoque » (79).

Le corps joue donc un rôle central dans le travail de purification et la mission de libération que s'assigne Léa. Effet purgatoire, car la musicienne crée chez ses spectateurs un bouleversement mystérieux qui leur permet de renouveler leur attitude envers eux-mêmes et envers les autres. Léa est décrite comme un personnage enthousiaste, toujours de bonne humeur, au cœur rempli de joie. Contrairement à sa mère, elle garde son sourire et son cœur d'enfant malgré toutes les terribles conditions qu'elle a vécues dans sa tendre enfance ${ }^{3}$. Femme douée pour le bonheur, elle se crée de et par son corps :

Peut-être est-ce sa sexualité qui passe dans sa musique et qui met le public dans un état de bonheur gavé, les mots ne le disent pas, bien sûr, mais les phrases musicales que compose Léa ont le pouvoir d'amener les hommes et les femmes qui les reçoivent à un point d'excitation extrême qu'ils ne nomment pas, et lorsque le concert se termine, ils sont tous satisfaits et comme exténués de délice sans comprendre qu'ils ont connu la jouissance (62-63).

\footnotetext{
${ }^{3}$ Petite enfant dans une cave pendant la guerre, puis adolescente dans un orphelinat à Berlin.
} 
Tout comme la musique, la sexualité est pour Léa un art qu'elle sait maitriser. Non seulement elle en jouit, mais elle réussit à concilier ces deux domaines qui paraissent de prime abord très éloignés. La musicienne offre son corps à tout homme qui entre dans sa vie et y laisse une trace particulière (David, Elie et Lucas). Chaque corps masculin rencontré offre à la compositrice une nouvelle source d'inspiration. Après chaque spectacle où elle «tire du violoncelle des sons voluptueux», Léa vit comme un soulagement indispensable son échange sexuel avec David, lequel «tire de son corps à elle un chant insoupçonné » (61). Et ce n'est nullement par hasard que la musicienne y trouve son bonheur puisque «l'accompagnateur a une science du sexe qui enchante son amie » (61). En effet, cette « science du sexe » est décrite en détail, lorsque les deux corps se donnent l'un à l'autre sans retenue; ainsi dans la scène-clé où les amants s'offrent des instants de plaisir sur la banquette arrière de la voiture qui les emmène à la sortie d'un concert.

Une écriture directe et fluide laisse entrevoir à partir de cette scène un acte sexuel poétisé. Bien que Lalande y emploie des termes qui renvoient au monde animalier - tels que "fourrure, oiseau, forêt, caverne », ce qui confère aux corps un aspect naturel -, la description de la scène érotique entre Léa et David est empreinte d'une profonde poésie. Car d'autres termes nettement plus poétiques - tels que "magique, trésor, étoilés, sensibles, douceur, secret, chant...»- viennent contrebalancer les termes quelque peu vulgaires (suce, sexe, liquide, clitoris). Ainsi deux registres opposés se conjuguent-ils subtilement pour dévoiler un corps en pleine jouissance, un moment dont Léa est la principale bénéficiaire. Ses expériences érotiques multiplient son désir de pénétrer son public - «... elle précisa dans un grand sourire qu'elle avait la volonté de pénétrer en chacun d'eux, oui, de les connaittre à la manière biblique » (63) -; elle déclare et affirme sa volonté de faire l'amour à son public - tout comme elle le fait aux hommes de sa vie. Cette conception musicale qui marie corps et composition marque l'objectif ultime de Léa, à savoir son désir de procurer chez ses spectateurs des sentiments orgasmiques d'euphorie et de sérénité. La musicienne joue pour donner du plaisir à son public, mais aussi pour l'éduquer : faire oublier aux hommes leurs fureurs et leurs cruautés. Dans ce sens, à travers son projet musical, l'épanouissement du corps de Léa affine la pensée de son public et le délivre de sa rudesse.

À partir d'une autre scène érotique, où Léa offre un spectacle privé à Elie en jouant au violoncelle toute nue, le corps acquiert une dimension poétique supplémentaire: "[Elie] s'est assis en face d'elle, il sourit, il la regarde, il la boit, il sait qu'elle joue la partition sur son corps à lui, qu'il est instrument aux mains de Léa » (82). L'instrument de musique y devient une métaphore du corps d'Elie que Léa exploite avec désir. Il s'agit là d'une mise 
en exergue du plaisir érotique puisque les deux personnages jouissent doublement: "elle tente de reculer le lâcher des étincelles d'amour, elle résiste, résiste, émue par le spectacle d'Elie qui murmure qu'il n'en peut plus, alors elle entame un lent crescendo, puis le violoncelle émet un son comme une longue plainte, le gémissement primitif du plaisir... » (82). Les amants atteignent deux fois le plaisir, le premier auditif et le second corporel. L'assimilation inhabituelle du violoncelle à un corps masculin (puisque c'est généralement l'inverse qui se produit) témoigne d'une grande fusion et d'une harmonie extrême entre la musicienne et son instrument. Son art musical s’identifie alors à un art érotique où Léa sait parfaitement mener le jeu. Le spectacle auquel Elie assiste et participe est à la fois un spectacle musical et érotique procuré par cette femme capable de le conduire vers la jouissance totale. Cette forme de plaisir délègue au corps une dimension musicale qui permet à Léa de mener à bien son projet artistique.

Avec Léa, le corps est non seulement un lieu de libération et d'épanouissement ainsi qu'une source de plaisir; il est aussi un passage nécessaire à l'accomplissement de sa musique et, en conséquence, se transforme en un instrument qui chante le plaisir. Pour Léa, parcourir le corps d'Elie, c'est comme parcourir les cordes d'un violoncelle, ou l'inverse. Cette osmose des corps crée une harmonie nouvelle où sexualité et musique se rejoignent. La nudité, le langage cru et brut sont vite écartés, ils deviennent synonymes de poésie quand l'amour transparaît entre les lignes. Le corps se détache alors du domaine de l'obscène pour se vêtir d'une nouvelle fonction qui le place au même rang que l'esprit : il devient verbe quand la parole ne suffit pas : «les mots ne le disent pas, bien sûr » (62). De ce fait, la fonction corporelle complète la fonction verbale. Les amants n'échangent que peu de paroles; ce sont leurs corps et leurs gestes qui se parlent. Le verbe n'est utile que pour servir l'acte sexuel : « Vas-y, serre! Desserre! Serre! » (61).

Dans Fragments d'un discours amoureux, Barthes conçoit celui-ci comme un discours théâtral. Théâtral, car il lui emprunte le drame, le va-et-vient permanent et la course de l'amoureux. L'énonciation du «je» amoureux s'identifie à celle du « je » propre au genre dramatique. Dire son amour, c'est comme prononcer un monologue sur une scène de théâtre. Dans le roman, l'amour en général et «l'amour du monde » en particulier sont développés essentiellement par le corps. Le discours est pratiquement absent dans toutes les scènes où le sentiment d'amour se manifeste. Le monologue amoureux se fait juste après un échange érotique, soit pour l'analyser, soit pour en partager l'intensité. L'expression de l'amour se fait davantage par les caresses et les attouchements du corps, ce qui nous amène à déduire que l'émotion est elle aussi théâtralisée, comme le déclare Barthes, mais elle l'est grâce à l'expression corporelle et non verbale. L'énonciation cède ici la place au langage charnel qui permet aux amants de communiquer leur bonheur. Corps et langage deviennent très proches et se complètent. La fonction érotique dans le roman est donc sublimée au détriment de la parole qui n'est 
prononcée que pour servir le corps «Ils nouaient leurs corps sans dire un mot, les sourires et la lumière des regards parlaient mieux que leurs voix » (33). Le «corps à corps» devient par lui-même une poésie au sens étymologique du terme. "Le langage est une peau » et la peau est un langage : le toucher, les caresses et la sensualité offrent au corps cette poésie dont il a été longtemps dépourvu. Ainsi, la sexualité des personnages est ce qui leur permet d'exprimer leurs sentiments. Le corps partagé avec l'autre devient une entité par laquelle les partenaires prennent définitivement conscience de leur existence.

Dans l'union charnelle, l'autre est mis à nu et exploré par le biais d'un échange qui offre aux amants le plaisir et leur procure un moment d'extase et de bonheur commun. Comme Léa le dit à David, "la sexualité est générosité » (62) : l'échange implique l'autre, tout comme la musique de cette jeune compositrice est le résultat d'une grande générosité, un acte où l'autre est invité à vivre le plaisir érotique à travers les notes musicales. Mais la nature de cet échange diffère. La fusion des corps des protagonistes et la communication profonde qui en résulte permet à chacun d'avouer son altérité au-delà du plaisir partagé. La générosité apparaît donc dans cette conception du corps qui cherche non seulement à s'épanouir, mais aussi à atteindre la jouissance tout en assumant l'autre-corps.

\section{Mythification et descente aux enfers : corps et souffrance}

L'expression «handicapés de l'amour » apparait plusieurs fois dans le roman. Ce que nous y observons, c'est un renouvellement perpétuel des expériences amoureuses. Les trois personnages principaux sont tous voués, dans leurs relations, soit à une déception, soit à une rupture voulue. C'est dire que chacun d'entre eux n'arrive pas à se retrouver complètement dans l'autre. Dès les premières pages du récit, la recherche d'un amant ou d'une amante s'avère une entreprise difficile ${ }^{4}$. Les trois personnages principaux multiplient les relations amoureuses, mais, à chaque fois, ils en sont insatisfaits ou déçus. Cette incapacité à aimer, cette impuissance à trouver dans le partenaire ce que l'on cherche, est annoncée comme une thématique intrinsèque à la progression de chacune des trois histoires. Le mot « handicapé » implique la notion d'inaptitude, d'invalidité à réaliser une tâche définie. Les personnages sont donc marqués par une déficience, une sorte d'infirmité mentale qui fait d'eux des êtres éternellement insatisfaits, non faits pour ce sentiment qui leur semble étrange, voire étranger. Victimes d'une histoire qui saigne, ils portent tous sur leur dos le poids de la souffrance vécue par leurs familles. L'impuissance d'aimer est en effet un

\footnotetext{
${ }^{4}$ Léa rompt avec Elie, puis rencontre Lucas en passant par David. Lila croit trouver l'homme de sa vie, Dostoïevski, se marie avec lui, avant de le quitter; elle rencontre Samuel avec qui elle pense vivre une relation assez particulière, mais elle s'en lasse et n'hésite pas à s'éloigner de lui. Julius, lui, trompe sa femme avec Rachel, puis découvre son homosexualité en couchant avec un marin; il s'enfuit et laisse tout derrière lui.
} 
lourd handicap qui se transmet comme un héritage de génération en génération. L'amour est assimilé à une maladie chronique que chaque personnage porte dans ses gênes. Léa, Lila et Julius sont des bossus de la dramatique histoire juive, tous les trois souffrent d'une malédiction ancestrale : membres de familles ayant subi la persécution nazie, ils gardent au plus profond d'eux-mêmes cette cicatrice qui les accompagnera tout au long de leur parcours. Ces trois descendants décrits comme des « rescapés de la folie assassine " mènent en effet une vie marquée par un passé douloureux qui pèse et ne s'oublie pas : «On croit effacer les erreurs passées, oublier les douleurs anciennes, comment peut-on être aussi naïf? (33).

Les blessures de l'histoire juive sont des souffrances dont nulle victime ne peut se détacher. Que ce soit en Allemagne, en Belgique ou aux États-Unis, la souffrance est indéniablement présente, quoique différente. Les parcours suivis par les trois protagonistes se présentent comme des quêtes destinées à trouver une paix intérieure, à se réconcilier avec soi-même et avec les autres. Si leur vie commence par une négation et des incertitudes qu'ils cherchent tant bien que mal à surmonter à travers leur enthousiasme (conditionné par le corps) et leur détermination, tous trois apprennent progressivement non seulement à supporter leur douleur, mais aussi à la dépasser en défiant le destin : le voyage qu'ils entreprennent en Israël afin d'y rencontrer leurs semblables apparait comme le couronnement de cette recherche et la preuve de leur ferme volonté de réconciliation avec soi et avec les autres. Mais le voyage qui nous intéresse le plus dans le cadre de cette étude, c'est celui de l'âme, celui qui est effectué aux enfers de l'inconscient, aux tréfonds de la mémoire blessée et blessante. En effet, le traçage des trois biographies commence par la description de leurs arbres généalogiques et de leurs naissances. Cette genèse et ce retour au passé lointain constituent l'essence même et le symbolisme du thème de la descente au royaume d'Hadès. Il s'agit bel et bien d'un retour aux origines pour y réaliser un rite initiatique indispensable. Un passage obligé, une étape indispensable afin que les personnages trouvent la voie nécessaire à leur (re) construction. Certes, c'est le narrateur qui déclenche ce retour et qui souligne ce commencement, mais les personnages eux-mêmes ne peuvent point s'empêcher de faire de temps à autre un saut rétrospectif dans leur passé. Dans ce contexte, on ne peut parler de l'Enfer sans penser aux personnages de la mythologie grecque et principalement à Ulysse qui s'engagea dans un long voyage et passa notamment par les enfers avant de pouvoir regagner Ithaque, son royaume. L'Odyssée relate le parcours d'un roi perdu dans un monde inconnu, celui d'un homme désireux de retrouver sa patrie et de revoir les siens. Pour chacune des épreuves auxquelles il dut faire face, Ulysse, ce héros d'une épopée marquée par la douleur du retour, trouva une solution. Dans ce "nostos algos», ce retour douloureux, Ulysse se perdit et erra de ville en ville, s'efforçant à chaque fois de surmonter les épreuves et de trouver le vrai chemin. Sa détermination résidait dans sa fidélité à ses origines et à sa mémoire. 
N'est-ce pas un même projet qui unit les trois personnages du roman lalandien au héros homérique? Deux mots mettent en exergue la ressemblance existant entre eux : voyage et souffrance. Comme Ulysse, Léa, Lila et Julius sont fidèles à eux-mêmes : leur origine ne les empêche point d'arriver au bout de leurs ambitions. Tous les trois vivent avec la cicatrice d'un passé familial atroce qui, même s'il est source de douleur constante, se doit d'être apprivoisé : « Nous ne guérirons jamais de nos douleurs, mais la vie que nous menons les apprivoise » (84), comme l'énonce Léa dans une conversation avec Elie. Dans cette fidélité à soi-même, Ulysse vit avec nostalgie l'absence de sa terre natale, de son royaume, de Pénélope et du milieu auquel il appartient; les personnages du roman lalandien, eux, vivent l'absence d'une histoire qui leur ferait justice ainsi que d'un monde compréhensif qui les accueillerait à bras ouverts; ils sont, eux aussi, en quête d'une patrie capable de les unir et de respecter leur différence.

Que ce soit dans l'un ou l'autre cas, le monde de l'errance et de la perdition est toujours présent. Afin de trouver le chemin du retour et de poursuivre son périple, Ulysse doit descendre aux enfers pour y interroger Tirésias, une épreuve qu'il surmonte avec succès. De même, dans la recherche de soi ainsi que dans leur quête destinée à trouver un accord avec eux-mêmes et avec le monde qui les entoure, les trois protagonistes du roman lalandien sont obligés de descendre aux enfers eux aussi, une descente qui consiste à explorer le douloureux passé de leurs proches.

Chaque expérience vécue contient intrinsèquement des épisodes qui forcent chaque protagoniste à effectuer un retour nostalgique, parfois même tragique, dans l'Histoire. L'Hadès de ces personnages constitue la mémoire, traduit par des analepses qui surgissent subitement pour leur rappeler leur condition. Ce retour vers le passé n'est autre que le retour vers l'inconscient, vers une souffrance enfouie, domptée par un corps qui lutte pour garder sa place dans un monde frappé d'amnésie. Dans ce monde cruel, absurde et meurtrier, l'homme a tendance à oublier les crimes qu'il a commis. Les personnages lalandiens, eux, n'oublient pas; leur présence et le combat qu'ils $\mathrm{y}$ mènent contre l'amnésie est en lui-même un témoignage formidable et réconfortant.

La seule différence qui existe entre ces trois personnages et Ulysse, c'est que tandis qu'ils cherchent à protéger leur mémoire, à témoigner de leur histoire afin qu'elle soit reconnue, le héros mythique, lui, s'applique à retrouver sa mémoire perdue. Sa descente aux enfers est concrétisée par un certain nombre de rituels qu'il effectue avant de rencontrer Tirésias. Léa, Lila et Julius voyagent, eux, aux enfers de leur propre histoire en passant par des rites comparables avec ceux du voyage d'Ulysse : de même qu'il accède au royaume d'Hadès afin d'y interroger le devin Tirésias sur son retour à Ithaque, le voyage des trois personnages s'affirme comme étant la recherche 
d'une reconnaissance, la possibilité d'un retour sans douleur ni souffrance. Dans les deux cas, cette double recherche de l'identité est conditionnée par le corps. "Ulysse, en revenant devient», selon l'expression de Florence Bronstein et Jean François Pépin dans La Place du corps dans la culture occidentale; son retour vers Ithaque forge sa quête mystique. Les expériences - surtout, les corporelles - que vivent les trois juifs marquent leur voyage vers un autre « je», celui qui trouve le bonheur que chacun mérite.

Ce thème de la quête mystique, fréquent dans la littérature universelle, répond fondamentalement à quatre étapes qu'énumèrent Bronstein et Pépin :

1) Un rite de séparation qui arrache le novice à son milieu: dans cette étape, l'être subit une rupture d'un certain rythme auquel il est habitué. Cette rupture est déclenchée soit par l'individu en personne soit par un élément extérieur qui l'oblige à se détacher de son entourage. Après la mort de Myriam-la-jeune, Léa se retrouve seule et orpheline; elle est accueillie dans un orphelinat dès son jeune âge. Lila, elle, de son plein gré, décide de quitter sa famille par sentiment d'étouffement et par volonté d'être libre. Julius, lui aussi, se détache de sa mère : l'excès d'amour et le désir de possession manifesté par celle-ci le poussent à quitter le cocon familial pour aller s'installer dans le bateau de son défunt père. Toutes ces séparations surviennent suite à des évènements qui marquent d'une certaine manière les personnages et constituent un point de départ vers une nouvelle vie, différente et plus périlleuse.

2) Un rite de marginalisation où survient avant la mort un certain nombre d'épreuves: les épreuves que vivent les héros du roman sont principalement des épreuves d'ordre corporel. Comme nous l'avons vu, le rapport de Léa au corps est assez particulier : en cherchant le bon partenaire, Léa se recherche elle-même. C'est avec David qu'elle découvre son corps et qu'elle apprend à l'interroger. Son rapport à Elie est un peu moins érotique: elle découvre le monde professionnel, apprend à écouter, à analyser et à débattre. Et c'est principalement avec Lucas qu'elle subit une épreuve douloureuse : son histoire passionnelle avec cet homme s'achève en effet sur une révélation choquante, celle de la relation amoureuse qu'adolescent, il vécut avec Myriam-la-jeune. Aussi leur union débouche-t-elle sur une séparation douloureuse pour Léa.

Lila, elle aussi, vit un certain nombre d'épreuves à travers son corps. De son mariage avec Dostoïevski, elle écrit un journal intime où elle tente de cicatriser les blessures du passé. Avec Samuel, elle goûte l'excès et la démesure; elle voit le monde - ou plutôt l'autre différemment: c'est ainsi, par exemple, qu'elle se détache de ses préjugés sur les Palestiniens en découvrant l'humour et l'honnêteté de Naïm Khader, le représentant de l'OLP à Bruxelles. Par la suite, 
elle renoue avec ses combats de lutteuse, accumule les victoires jusqu'à ce qu'elle décide de tout arrêter.

Quant à Julius, il se recherche non pas par le biais de son corps, mais plutôt dans son corps. Avec sa première femme, il expérimente la beauté, l'innocence, et vit l'amour pur. Avec Rachel, la femme forte, moche et brutale, il vit tout le contraire de ce qu'il a connu avec Deborah. Son insatisfaction avec l'une et l'autre le mène à la découverte de son homosexualité à l'occasion d'une rencontre avec un inconnu dans un bar...

La marginalisation de ces trois personnages réside dans le fait qu'ils sont uniques, chacun ayant sa propre histoire. Chacun d'eux porte en lui un objectif particulier : retrouver le bonheur qu'il mérite, un bonheur certes difficile à conquérir, qui leur sera offert d'une certaine façon au bout de leur quête.

3) Un rite de renaissance ou de reconnaissance: dans cette phase, les personnages commencent à y voir plus clair. Sachant qu'aucune des épreuves par lesquelles ils sont passés, ne donne le vrai bonheur, ils décident de répondre positivement à la lettre qui les invite à une réunion de la famille Keil, organisée à Tel-Aviv. Ce voyage commun permet aux trois personnages de se retrouver dans un même lieu, où chacun se reconnait dans l'autre. La recherche du bonheur perdu aboutit donc à cette union fraternelle qui rassemble trois âmes jumelles dans leur souffrance et dans leur perdition.

4) Un rite d'initiation rendant possible le retour au monde réel: les voyages de Léa, Lila et Julius se croisent en Israël, là où ils se rencontrent et où trouvent une réponse commune à leur quête individuelle. La lettre qu'ils laisseront à l'enquêteur qui les découvrira constituera le fruit de cette longue recherche de l'amour et du bonheur révolu. La fin du voyage, si elle rend impossible le retour au monde réel, leur ouvre une nouvelle brèche. La mort comme choix porte en elle des significations pouvant être lues comme une mise à mort symbolique de toutes les injustices dont ils ont souffert.

Cette ultime expérience permet aux héros d'arriver à un point d'ascension symbolisé par le partage, la compassion et la compréhension qu'ils ont tant recherchées et que chacun d'eux ressent chez l'autre. L'initiation se fait par la mort finale des héros qui, en dépit et à l'issue de toutes les épreuves endurées - déceptions, souffrances et désillusions -, trouvent leur bonheur et une paix intérieure. Ces trois héros humains et humanistes renoncent donc à un monde où l'homme succombe fréquemment à sa laideur, à ses pulsions meurtrières, en emportant avec eux leurs valeurs longtemps recherchées et retrouvées au terme de leur long voyage dans l'espace et dans le temps. Ainsi la quête mystique dont nous avons parlé plus haut trouve-t-elle son apogée dans cette mort douce qui confère toute sa signification à cette métaphore du voyage. 


\section{Conclusion}

Le parcours des personnages se présente ainsi comme une sorte d'épopée, un voyage où les vagues de la mémoire sont aux héros ce que le chant des sirènes est pour Ulysse. La puissance incontrôlable qui les meut dans cette entreprise de recherche de soi est souvent doublée par la sensation d'être soumis à une force inconnue, «la douloureuse sensation qu'une main inconnue fermait une porte derrière elle » (35). De même qu'Ulysse se laisse diriger par les dieux de l'Olympe, les personnages lalandiens sont guidés par une main inconnue qui tente de les soustraire à cette "malédiction ancestrale ».

Voyager pour découvrir, s'ouvrir à l'autre et essayer de se faire de nouvelles idées. Voyager pour apprendre, s'initier et partir à la recherche de nouvelles significations dans le monde de l'inconnu. Le sentiment permanent de vouloir aimer avec force est le résultat de cette blessure héritée et face à laquelle nos trois héros se placent comme des individus désireux de changer, de défier et de reconstruire ce qui a été anéanti. D’une certaine manière, tous les personnages lalandiens, principaux ou secondaires, font partie d'une histoire qu'il s'agit de relater afin qu'elle ne tombe pas dans l'oubli. Le point commun qui les unit, c'est un corps qui souffre, mais qui paradoxalement s'épanouit, un leitmotiv incontournable autour duquel tournent toutes leurs histoires. 


\section{Bibliographie}

Barthes, Roland. Fragments d'un discours amoureux. Paris : Cérès, 2005.

Bloch, Béatrice. Le roman contemporain: liberté et plaisir du lecteur. Paris: L'Harmattan, 1998.

Braunstein, Florence et Pépin, Jean-François. La place du corps dans la culture occidentale. Paris : Presses Universitaires de France, 1999.

Dadoun, Roger. L'Erotisme. De l'obscène au sublime. Paris: Presses Universitaires de France, 2010.

Gaspard, Jean-Luc et Doucet, Caroline (dir.). Pratiques et usages du corps dans notre modernité. Paris : Erès, 2009.

Lalande, Françoise. Nous veillerons ensemble sur le sommeil des hommes. Avin : Luce Wilquin, 2012.

Laporte, Philippe. L'érotisme ou le mensonge de Freud. Refondation de la psychologie sexuelle. Paris : Éditions Connaissance et savoir, 2009.

Marzano, Michela. Penser le corps. Presses Universitaires de France, 2002.

Paque, Jeannine. «Françoise Lalande et le devoir de résistance ». Interview par Jeannine Paque. Le carnet et les instants, no 171, 2012:11-14.

Sagini-Lebas, Yvette. Eléments d'érotique du texte. Paris : L'Harmattan, 2013.

Zupancic, Metka. Les écrivaines contemporaines et les mythes. Paris: Karthala, 2013. 